\title{
Low Pressure Photolysis of 2,3-Pentanedione in Air: Quantum Yields and Reaction Mechanism
}

\author{
Hichem BOUZIDI ${ }^{1,2}$, Mokhtar DJEHICHE ${ }^{1,29}$, Tomasz GIERCZAK ${ }^{3}$, Pranay \\ MORAJKAR $^{4 \#}$, Christa FITTSCHEN ${ }^{4}$, Patrice CODDEVILLE $^{1,2}$, Alexandre TOMAS ${ }^{1,2 *}$
}

${ }^{1}$ Mines Douai, SAGE, 59508 Douai, France

${ }^{2}$ Université de Lille, 59000 Lille, France

${ }^{3}$ Faculty of Chemistry, Warsaw University, ul. Pasteura 1, Poland

${ }^{4}$ Université de Lille 1, PC2A, UMR 8522 CNRS/Lille 1, 59655 Villeneuve d'Ascq, France

${ }^{\$}$ Current address: University M'Sila, Faculty of Sciences, M'Sila, Algeria

${ }^{\#}$ Current address: Goa University, Department of Chemistry, Taleigao 403206, Goa, India

*: corresponding author:

Prof. Alexandre Tomas

Département Sciences de l'Atmosphère et Génie de l'Environnement, Ecole Nationale Supérieure des Mines de Douai, 941 rue Charles Bourseul, CS 10838, F-59508 Douai Cedex, France alexandre.tomas@mines-douai.fr

Tel.: 00.33.3.27.71.26.51

Fax: 00.33.3.27.71.29.14

Supplementary Information 
Figure S1. PTD (solid curve) ${ }^{1}, \mathrm{NO}_{2}$ (doubled curve) ${ }^{2}$ absorption spectrum and lamp emission spectrum (dotted curve). The vertical stretched line indicates the highest PTD photodissociation threshold $(400 \mathrm{~nm})$.

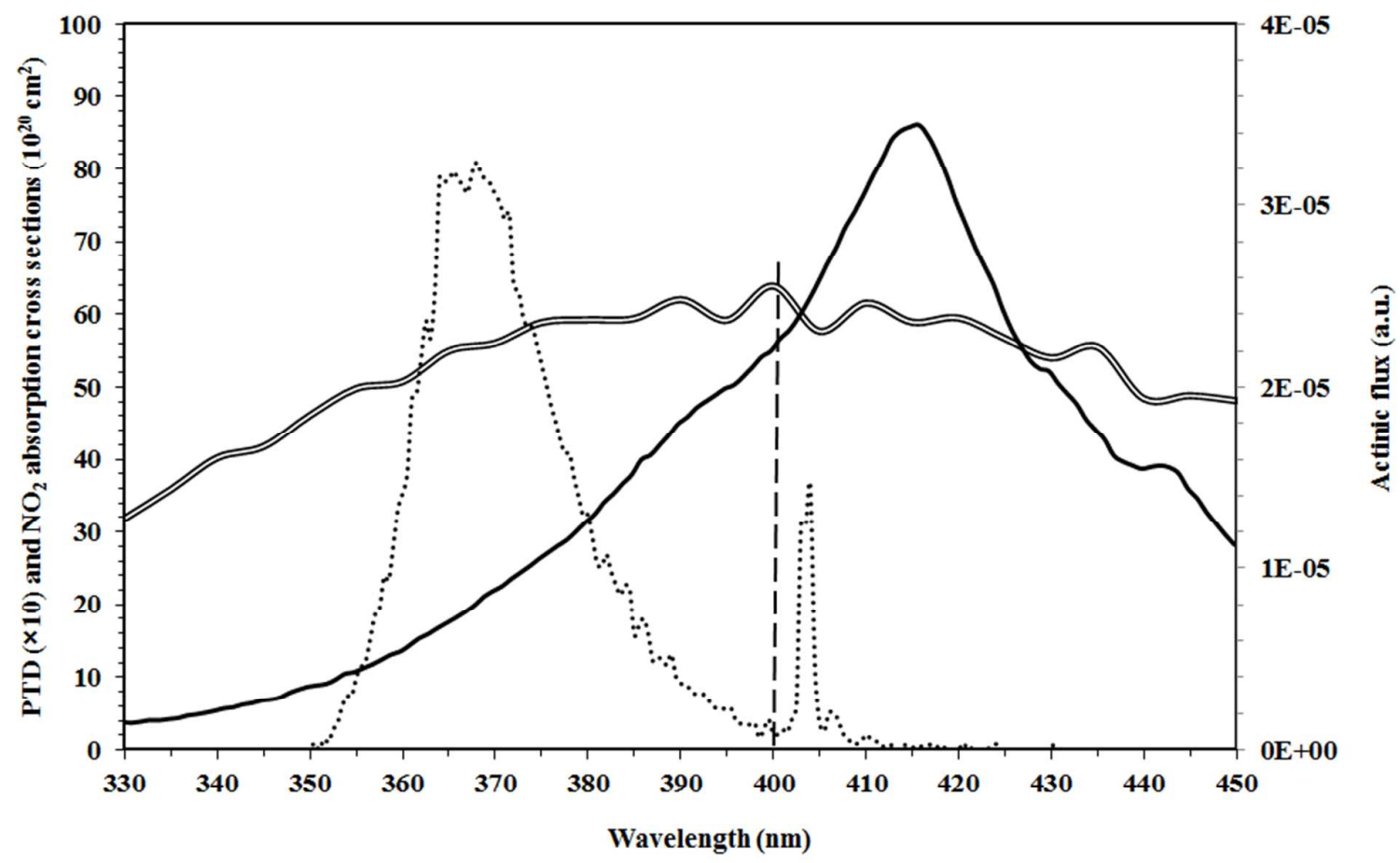

(1) Szabo, E.; Djehiche, M.; Riva, M.; Fittschen, C.; Coddeville, P.; Sarzynski, D.; Tomas, A.; Dobé, S., Atmospheric chemistry of 2,3-pentanedione: Photolysis and reaction with $\mathrm{OH}$ radicals. $J$. Phys. Chem. A 2011, 115, 9160-9168.

(2) Atkinson, R.; Baulch, D. L.; Cox, R. A.; Crowley, J. N.; Hampson, R. F.; Hynes, R. G.; Jenkin, M. E.; Rossi, M. J.; Troe, J., Evaluated kinetic and photochemical data for atmospheric chemistry: Volume $\mathrm{I}$ - gas phase reactions of $\mathrm{O}_{\mathrm{x}}, \mathrm{HO}_{\mathrm{x}}, \mathrm{NO}_{\mathrm{x}}$ and $\mathrm{SO}_{\mathrm{x}}$ species. Atmos. Chem. Phys. 2004, 4, 1461-1738. 